\title{
Observation of incident angle dependent phonon absorption in hydrogenated amorphous silicon nitride thin films
}

\author{
Tong Li and Jerzy Kanicki ${ }^{\mathrm{a})}$ \\ Center for Display Technology and Manufacturing, Department of Electrical Engineering and Computer \\ Science, The University of Michigan, Ann Arbor, Michigan 48105
}

(Received 18 February 1998; accepted for publication 23 October 1998)

\begin{abstract}
We have demonstrated the existence of longitudinal- and transverse-like optical modes of $\mathrm{Si}-\mathrm{N}$ bond in vibrational absorption spectrum of hydrogenated amorphous silicon nitride thin films. One of the longitudinal-like optical resonances coincides with the transverse-like mode of $\mathrm{Si}-\mathrm{O}$ bond, and the other closely neighbors the bending mode of $\mathrm{N}-\mathrm{H}$ bond. We have also shown that the conventionally assigned asymmetric stretching mode of $\mathrm{Si}-\mathrm{N}$ bond is merely a transverse-like mode of the bond. The microstructures of both longitudinal- and transverse-like modes can well be apprehended by a p-polarized beam at an oblique incidence light, especially at Brewster angle incidence. The spectrum distortion induced by interference fringes can be eliminated at this condition. (C) 1998 American Institute of Physics. [S0003-6951(98)04452-0]
\end{abstract}

Hydrogenated amorphous silicon nitride $\left(a-\mathrm{SiN}_{x}: \mathrm{H}\right)$ thin films have attracted much attention, ${ }^{1,2}$ mainly because of their indispensable role in amorphous silicon thin-film transistor $(a-\mathrm{Si}: \mathrm{H}$ TFT) structures as a superior gate insulator. Knowledge of their microstructures and the electron-phonon scattering processes is very essential for making a reliable electronic device based on such materials. In this letter, we report the observation of incident angle dependent phonon absorption in vibrational absorption spectrum of $a-\mathrm{SiN}_{x}: \mathrm{H}$ by using Fourier transform infrared spectroscopy (FTIR). ${ }^{3,4}$ We have found that the longitudinal-like optical (LO) vibrational modes associated with $\mathrm{Si}-\mathrm{N}$ bonds are located in the spectrum region that overlaps with some well-known bonds such as transverse mode (TO) of $\mathrm{Si}-\mathrm{O}$ and bending mode of $\mathrm{N}-\mathrm{H}$. Therefore, distinguishing those modes (bonds) is very essential in $a-\mathrm{SiN}_{x}: \mathrm{H}$ film's quality assessment.

The $a-\mathrm{SiN}_{x}: \mathrm{H}$ thin films studied in this work were prepared by plasma-enhanced chemical vapor deposition (PECVD) on double-side polished Si wafers. The film depositions were carried out at the process chamber pressure of 0.43 Torr, the radio frequency (rf) power of $100 \mathrm{~W}$, the substrate temperature of $250^{\circ} \mathrm{C}$, and ammonia to silane flow ratio of 200/18. Following the deposition, the film's thickness was measured using Dektak surface profiler, while the film's refractive index was deduced by observing a straight and horizontal baseline at Brewster incident angle with p-polarized light. ${ }^{5}$ The film's dielectric constant at low frequency $(1 \mathrm{MHz})$ was derived from capacitance-voltage $(C-V)$ characteristics based on the metal-insulatorsemiconductor (MIS) structure. The stoichiometry and hydrogen content ( $\mathrm{H}$ content) of the film are determined by Rutherford-backscattering spectroscopy (RBS). Hydrogen evolution ( $\mathrm{H}$ evolution) was carried out by the thermal annealing at $600{ }^{\circ} \mathrm{C}$ under vacuum in Leybold sputtering system. The IR spectra were recorded at room temperature using Bio-Rad FTS-40 Fourier transform infrared spectrometer and analyzed with Bio-Rad Win-IR software. The aperture of

a)Electronic mail: kanicki@eecs.umich.edu probe beam and the detector gain amplification were set at 2 $\mathrm{cm}^{-1}$ and unity, respectively. Meanwhile, the $p$-polarized probe beam was produced by a pike $\mathrm{ZnSe}$ wire grid linear polarizer, which was adjusted to an appropriate incident angle by a Harrick Brewster's angle sample holder. The resultant spectrum having a spectral resolution of $8 \mathrm{~cm}^{-1}$ is an average of 64 scans.

It was first reported by Berreman in 1963 that the longitudinal optical mode of the thin film of cubic crystal strongly absorbs $p$-polarized light at oblique incidence. ${ }^{6}$ Since then many similar observations of the TO-LO pairs in amorphous materials have been reported. ${ }^{7-9}$ According to Berreman's theory, a thin film with cubic or cubic-like structure has two normal modes of polarized lattice vibrations. One of the vibrations is parallel to the film surface with its frequency of $\omega_{t}$, while the other is normal to the film surface with its frequency of $\omega_{1}$. The former produces no electric field and occurs when the dielectric constant is very large. On the other hand, the latter produces an electric field normal to the surface due to the surface polarization and occurs when the dielectric constant is minimized. If an electric field parallel to the film surface with its frequency near $\omega_{t}$ is incident upon the film, lattice vibrations parallel to the surface will have a large amplitude due to a resonance phenomenon, since internal and external components of electric field parallel to the film surface must match. Likewise, an electric field normal to the film surface with its frequency near $\omega_{1}$ will result in a large amplitude lattice vibrations normal to the surface, since the internal and external components of electric displacement normal to the surface must match.

When a $p$-polarized light beam strikes film with an oblique incidence, as illustrated in Fig. 1, the $p$ vector can be treated as the sum of vectors normal $\left(p_{\perp}\right)$ and parallel $\left(p_{\|}\right)$ to the film surface. Apparently, $p_{\perp}$ is responsible for LO mode resonance while $p_{\|}$attributes to TO mode resonance. The intensities of the LO and TO resonances are proportional to the magnitudes of $p_{\perp}$ and $p_{\|}$vectors, respectively. As indicated in Fig. 1, at normal incidence, $p_{\perp}$ vector vanishes and no LO-like modes can be observed in the vibrational 


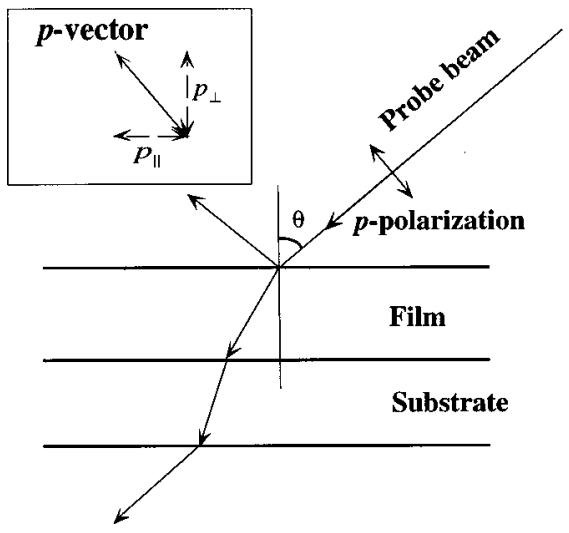

FIG. 1. Illustration of $p$-polarized light beam incidence on thin film at an oblique angle.

absorption spectrum. With the increase of light incident angle $\theta, p_{\perp}$ increases and $p_{\|}$decreases. Consequently, an increase of LO-like and a decrease of TO-like peak densities should be observed in vibrational absorption spectrum.

Figure 2 compares the vibrational absorption spectra of $a-\mathrm{SiN}_{x}: \mathrm{H}$ collected at various incident angles with $p$-polarized beam. When the beam incident angle increases from $0^{\circ}$ to $75^{\circ}$, the peak of so-called asymmetric stretching mode of $\mathrm{Si}-\mathrm{N}$ bond ${ }^{10,11}$ located at $880 \mathrm{~cm}^{-1}$ shrinks. In contrast, the shoulder of that peak evolves into another peak at $1040 \mathrm{~cm}^{-1}$, which is the wave number conventionally assigned to $\mathrm{Si}-\mathrm{O}$ (TO) bond. ${ }^{12,13}$ In addition, the peak assigned to bending mode of $\mathrm{N}-\mathrm{H}[\mathrm{N}-\mathrm{H}(b)]$ bond located at 1180 $\mathrm{cm}^{-1}$ is significantly intensified, ${ }^{11,14}$ and its location is slightly shifted to $1215 \mathrm{~cm}^{-1}$. Meanwhile the densities of the peaks assigned to stretching mode of $\mathrm{N}-\mathrm{H}[\mathrm{N}-\mathrm{H}(s)]$ and $\mathrm{Si}-\mathrm{H}[\mathrm{Si}-\mathrm{H}(s)]$ bonds, and bending mode of $\mathrm{N}-\mathrm{H}_{2}$ $\left[\mathrm{N}-\mathrm{H}_{2}(b)\right]$ bond remain relatively constant with increasing incident angle, although the peak shapes are distorted by interference fringes. ${ }^{15}$ The observation suggests that the bond associated with these decreasing and increasing absorption peaks contains longitudinal-like components of infrared-

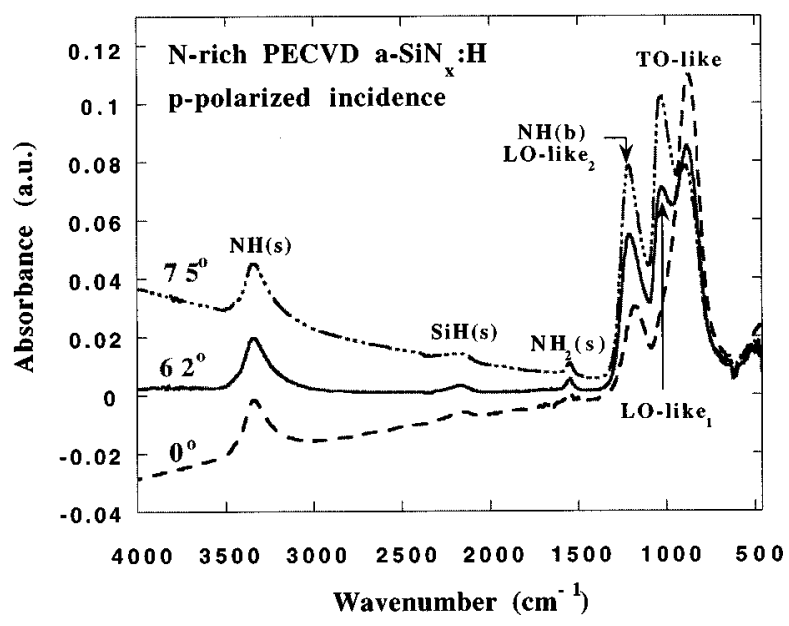

FIG. 2. Vibrational absorption spectra of N-rich PECVD $a-\mathrm{SiN}_{x}: \mathrm{H}$ thin film collected with $p$-polarized light beam incidents at $0^{\circ}, 62^{\circ}$ (Brewster angle), and $75^{\circ}$, respectively. The thickness and refractive index of the film are $1500 \AA$ and 1.88 , correspondingly. The scripts of $s$ and $b$, in the parentheses stand for stretching, bending modes, respectively, while LO-like stands for longitudinal-like mode and TO-like represents transverse-like optical modes of $\mathrm{Si}-\mathrm{N}$ bond. active vibrations which are sensitive to long-range electric forces. Thus, these decreasing and increasing peaks can be labeled as TO- and LO-like modes in $a-\mathrm{SiN}_{x}: \mathrm{H}$, respectively. The observed variation of the optical densities with $p$-polarized light at oblique incidence clearly indicates that the conventionally assigned asymmetric stretching mode of $\mathrm{Si}-\mathrm{N}$ bond located at $880 \mathrm{~cm}^{-1}$ is, in fact, only a TO-like resonance of that mode. In addition, the association of LOlike peaks with this TO-like resonance is suggested by the fact that $\mathrm{Si}-\mathrm{N}$ (TO like) mode is the only peak decreasing with the increasing incident angle, since LO- and TO-like modes are normally paired to each other in the absorption spectrum. Theoretically, frequency of LO phonons, $\omega_{l}$, is related to that of TO phonons, $\omega_{t}$, by the Lyddane-SachsTeller relation: ${ }^{6,16}$

$$
\frac{\omega_{l}^{2}}{\omega_{t}^{2}}=\frac{\epsilon_{0}}{\epsilon_{\infty}},
$$

where $\epsilon_{0}$ and $\epsilon_{\infty}$ are the dielectric constants at low and high frequency, respectively.

According to Eq. (1), the frequencies of LO modes should be always greater than those of TO modes, since $\epsilon_{0}$ $>\epsilon_{\infty}$, which is consistent with current observation. In particular, frequencies of both LO-like modes are greater than that of TO-like mode, but smaller than or equal to those of all other absorption modes of different bonds. This fact implies that the LO-like modes are only pairing with TO-like mode of $\mathrm{Si}-\mathrm{N}$ bond and are irrelevant to other bonds. The frequency relation between 1215 and $800 \mathrm{~cm}^{-1}$ absorptions can even be related numerically by using measured values of $\epsilon_{\infty}$ (3.53) and $\epsilon_{0}(6.8)$ in Eq. (1) $(1215 / 880 \approx \sqrt{6.80 / 3.53})$. These LO-like modes may be tentatively labeled as LO-like ${ }_{1}$ and LO-like ${ }_{2}$ modes as indicated in Figs. 2, 3, and 4. Note that in order to present and compare vibrational absorption spectra free of the distortion induced by interference fringes, all spectra illustrated hereafter were collected at Brewster angle with only $p$-polarized beam incidence unless otherwise indicated. ${ }^{15}$

To further confirm the conclusion drawn above, $a-\mathrm{SiN}_{x}: \mathrm{H}$ film was thermally annealed at $600^{\circ} \mathrm{C}$ for $20 \mathrm{~min}$. The comparison of the spectra before and after thermal annealing is shown in Fig. 3. It is well established that upon thermal annealing, hydrogen evolves out from the film and leaves behind the $\mathrm{Si}-$ and $\mathrm{N}$-dangling bonds. These $\mathrm{Si}$ - and $\mathrm{N}$-dangling bonds passivate each other to form a new $\mathrm{Si}-\mathrm{N}$ bond. This will result in an enhanced density of $\mathrm{Si}-\mathrm{N}$ bond and reduced densities of all hydrogen related bonds in the absorption spectrum. ${ }^{17,18}$ In Fig. 3, the peak amplitudes of both TO- and LO-like ${ }_{1}$ modes have increased after thermal annealing indicating their association with $\mathrm{Si}-\mathrm{N}$ bond. On the other hand, the optical densities of all other modes have been reduced with the exception of the peak located at 1215 $\mathrm{cm}^{-1}\left(\mathrm{LO}^{-} \mathrm{like}_{2}\right)$. If this peak only represented hydrogen related bond $[\mathrm{N}-\mathrm{H}(b)]$ as assigned conventionally, its amplitude would be reduced upon thermal annealing. But instead, its amplitude is enhanced after $\mathrm{H}$ evolution indicating that the density reduction of $\mathrm{N}-\mathrm{H}(b)$ mode is overcome by the density enhancement of another bond. In $a-\mathrm{SiN}_{x}: \mathrm{H}$ films, the only enhanced density after $\mathrm{H}$ evolution is that of $\mathrm{Si}-\mathrm{N}$ bond as indicated in a previous study. ${ }^{17}$ Therefore, it is le- 


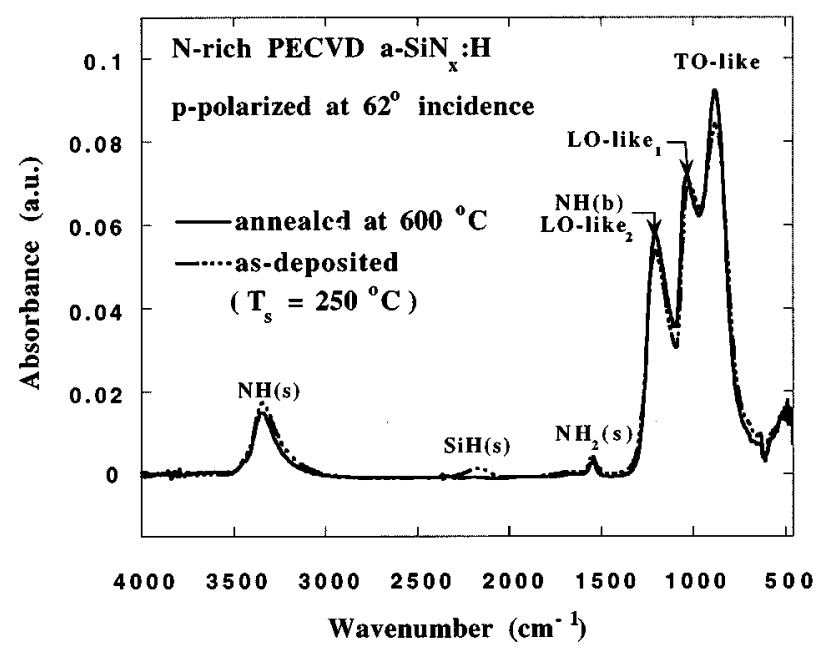

FIG. 3. Vibrational absorption spectrum comparison between as-deposited and after thermal annealing (at $600{ }^{\circ} \mathrm{C}$ for $20 \mathrm{~min}$ ) $a$ - $\mathrm{SiN}_{x}: \mathrm{H}$ thin films. Spectra were collected at Brewster angle with $p$-polarized beam. The thickness and refractive index of the film are $1500 \AA$ and 1.88 , respectively.

gitimate to conclude that one of the LO-like modes, which is pairing with the TO mode of $\mathrm{Si}-\mathrm{N}$, contributes to the optical density of $1215 \mathrm{~cm}^{-1}$ absorption. Moreover, comparison between the absorption spectra of films with a similar stoichiometry and thickness but different $\mathrm{H}$ contents shows a very similar trend (not shown), which is also consistent with the suggested TO-LO identification.

The absorption spectra of the films with very similar thicknesses and $\mathrm{H}$ contents but different stoichiometries are given in Fig. 4. The film with atomic composition closer to stoichiometry exhibits larger TO-like and LO-like 1 peak densities, since it contains a larger $\mathrm{Si}-\mathrm{N}$ bond density than other films. Meanwhile, the density of $1215 / 1240 \mathrm{~cm}^{-1}$ absorption is proportional to nitrogen content ( $\mathrm{N}$ content) of the film, which is the direct result of the proportionality between the $\mathrm{N}$ content and $\mathrm{N}-\mathrm{H}(b)$ density in the film. However, com-

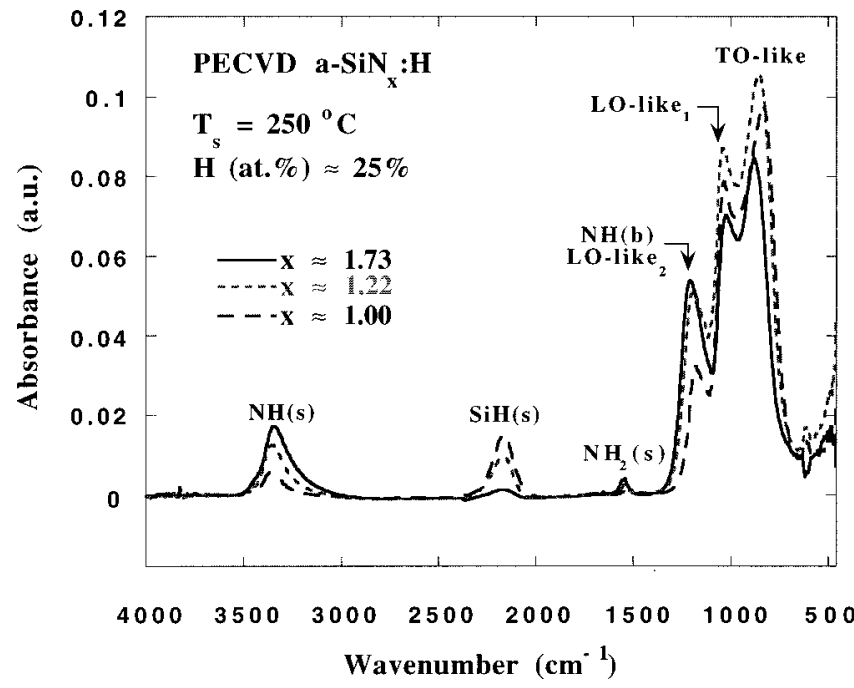

FIG. 4. Vibrational absorption spectrum comparison among films with the same thickness but different stoichiometries. The stoichiometry is represented by atomic ratio $x=\mathrm{N} / \mathrm{Si}$. Spectra were collected at Brewster angle with $p$-polarized beam. The thickness of the film is $1500 \AA$. The refractive indices of films with $x$ of $1.00,1.22$, and 1.73 are 2.00, 1.95, and 1.88, respectively. parison of this peak among the spectra with different atomic ratios shows that the density difference for $x$ of 1.73 and 1.22 is very small, while this difference is significantly large between $x$ of 1.73 and 1.00 as well as between $x$ of 1.22 and 1.00. According to the suggested relation between TO- and LO-like modes, the 1215/1240 absorption density should be proportional to the densities of $\mathrm{N}-\mathrm{H}(b)$ ( $\mathrm{N}$ content) and $\mathrm{Si}-\mathrm{N}$. When the film's composition changes from N- to Sirich, density of $\mathrm{N}-\mathrm{H}(b)$ decreases monotonically; while $\mathrm{Si}-\mathrm{N}$ density increases first, reaches maximum at stoichiometry, and starts to decline beyond the stoichiometry. Namely, the densities of $\mathrm{N}-\mathrm{H}(b)$ and LO-like 2 modes have an opposite trend within $\mathrm{N}$-rich to stoichiometry range, and have the same trend within Si-rich to stoichiometry range. This relation is reflected by the variation of LO-like ${ }_{2}$ absorption as a function of film's atomic ratio in Fig. 4.

In conclusion, we have observed the longitudinal-like modes in the vibrational absorption spectrum of $a-\mathrm{SiN}_{x}: \mathrm{H}$ thin films, and suggested their association with the transverse mode counterpart. The LO-like ${ }_{1}$ and $\mathrm{LO}^{-}$like $_{2}$ modes are located at about 1040 and $1215 \mathrm{~cm}^{-1}$, respectively, while TOlike mode is located at $880 \mathrm{~cm}^{-1}$ in the FTIR spectrum of $a-\mathrm{SiN}_{x}: \mathrm{H}$ film. It should be noted that the LO-like overlaps with TO-like mode of Si-O $\left(1040 \mathrm{~cm}^{-1}\right)$, and the LO-like 2 shares the same frequency range with the bending mode of $\mathrm{N}-\mathrm{H}$ bonds $\left(1180 \mathrm{~cm}^{-1}\right)$. Furthermore, the conventionally assigned asymmetric stretching mode of $\mathrm{Si}-\mathrm{N}$ bond is merely a transverse-like optical mode of the bond.

This work was supported by AFOSR/ARPA through the Multidisciplinary University Research Initiative (MURI) under the Contract No. F49020-95-1-0524, and by the Center for Display Technology and Manufacturing at the University of Michigan. The RBS measurement was carried out at the Michigan Ion Beam Laboratory at the University of Michigan.

${ }^{1}$ F. Finger, U. Kroll, V. Viret, A. Shah, W. Beyer, X. M. Tang, J. Weber, A. Howling, and Ch. Hollenstein, J. Appl. Phys. 71, 5665 (1992).

${ }^{2}$ A. Piccirillo and A. L. Gobbi, J. Electrochem. Soc. 137, 3910 (1990).

${ }^{3}$ S. Hasegawa, L. He, Y. Amano, and T. Inokuma, Phys. Rev. B 48, 5315 (1993)

${ }^{4}$ I. J. Auer, R. Meisels, and F. Kuchar, Infrared Phys. Technol. 38, 223 (1997)

${ }^{5}$ E. Hecht, Optics, 2nd ed. (Addison-Wesley, Reading, MA, 1989), Chap. 8

${ }^{6}$ D. W. Berremen, Phys. Rev. 130, 2193 (1963).

${ }^{7}$ C. T. Kirk, Phys. Rev. B 38, 1255 (1988).

${ }^{8}$ J. F. Scott and S. P. S. Porto, Phys. Rev. B 161, 903 (1967).

${ }^{9}$ K. B. Koller, W. A. Schmidt, and J. E. Butler, J. Appl. Phys. 64, 4704 (1988).

${ }^{10}$ S. Hawegawa, M. Matsuda, and Y. Kurata, Appl. Phys. Lett. 57, 2211 (1990).

${ }^{11}$ G. Lucovsky, J. Yang, S. S. Chao, J. E. Tyler, and W. Czubatyi, Phys. Rev. B 28, 3234 (1983).

${ }^{12}$ P. Lange, J. Appl. Phys. 66, 201 (1989); P. Lange, U. Schnakenberg, S. Ullerich, and H. J. Schliwinski, J. Appl. Phys. 68, 3532 (1990).

${ }^{13}$ P. H. Gaskell and D. W. Johnson, J. Non-Cryst. Solids 20, 171 (1976).

${ }^{14}$ J. Kanicki and P. Wagner, Electrochem. Soc. Proc. 87-10, 261 (1987).

${ }^{15} \mathrm{~T}$. Li and J. Kanicki, J. Appl. Phys. (to be published).

${ }^{16}$ R. H. Lyddane, R. G. Sachs, and E. Teller, Phys. Rev. 59, 673 (1941).

${ }^{17}$ T. Li, J. Kanicki, M. Fitzner, and W. L. Warren, Proceedings of AMLCDs '95, Lehigh, p. 123.

${ }^{18}$ W. L. Warren, J. Robertson, and J. Kanicki, Appl. Phys. Lett. 63, 2685 (1993). 